\title{
VARIA
}

\section{HISTOIRE DE LA TÉLÉVISION, GENRES ET PROGRAMMATION. LES PROGRAMMES POLITIQUES TÉLÉVISÉS GRECS}

\author{
Ioanna Vovou ${ }^{1}$
}

Ce travail vise à mettre en évidence l'émergence et la consolidation du débat politique, ainsi que le poids de l'histoire politique dans l'évolution de la télévision en Grèce, inscrites dans un contexte historico-politique précis, qui caractérise la télévision hellénique dès sa naissance. Dans cette perspective, la réflexion sur l'évolution de l'énonciation télévisuelle selon des règles qui semblent être cristallisées dans des schémas conceptuels ayant une valeur généralisable met en évidence le besoin d'une approche historique afin de localiser les éléments de la réalité avec lesquels la théorie sera mise en relation et en épreuve. Pour ce faire, le premier fil à suivre pour aborder et mettre en contexte l'évolution de la télévision grecque est intimement lié à la naissance du média en grande partie sous un régime dictatorial. Quelle évolution pour un média dont les conditions de création s'inscrivent dans un contexte politique tourmenté et démocratiquement déficitaire?

1 Maître de Conférences à l'Université Paris 13, Membre du CEISME/Paris 3Sorbonne Nouvelle

Recherches en communication, $\mathrm{n}^{\circ} 25$ (2006). 
Faisant échos des mutations de la télévision grecque, nous entreprenons une analyse de l'évolution des programmes politiques, inscrits dans la grille des chaînes, témoignant, à leur tour, de la relation étroite entre pouvoir politique et télévision, et ceci dans un paysage audiovisuel déréglementé à partir du début des années 90. La problématique relative aux rapports entre politique et télévision, ensemble avec celle des enjeux économiques divers de la télévision privée, dépassent les frontières nationales d'un pays, même si elles se posent en fonction des spécificités de chaque culture nationale. Ainsi, l'analyse de l'évolution des débats politiques à la télévision grecque vise à doter d'une dimension différente les discours insistant sur les relations de la télévision au pouvoir au niveau international et offre une grille de lecture et des éléments de comparaison avec les télévisions d'autres pays.

\section{«Genre télévisuel » et contexte de programmation}

Le choix d'étudier les évolutions de la télévision à travers les genres ne relève pas du hasard. La notion de genre issue des théories de la littérature, est centrale dans la perception et la compréhension des programmes par les récepteurs. Le contexte cognitif dans lequel est situé un programme télévisuel, ainsi que les hypothèses et les interprétations que le téléspectateur formule, dépendent du genre. ${ }^{1}$ François

1 Robert Scholes se référant aux propos de Hirsch note : «...Dans le monde des adultes, des erreurs de lecture très graves, portant sur des textes littéraires, ainsi que la plupart des erreurs de jugement critique, sont imputables à une mauvaise compréhension des genres chez le lecteur ou le critique. Dans son livre Validity in Interpretation (New Haven, 1967, p. 74), E. D. Hirsch défend de manière convaincante l'idée que 'la conception générique préliminaire qu'un interprète se fait d'un texte donné, est constitutive de tout ce qu'il comprend de ce texte par la suite, et qu'il en est ainsi tant que cette conception générique n'est pas modifiée'. Hirsch insiste sur le fait que le contexte dans lequel on lit le langage d'une oeuvre littéraire est générique. Dès qu'on commence à lire, on formule telle ou telle hypothèse relative au genre, qu'on affine à mesure qu'on avance dans sa lecture et qu'on cerne de toujours plus près la nature unique de l'oeuvre en question en repérant les affinités qu'elle entretient avec d'autres oeuvres qui utilisent le langage de la même manière. ». (Scholes, 1986 : 79).

De même, F. Jost se réfère aux modes d'énonciation télévisuelle (authentifiant, fictif, ludique) et insiste sur le fait «que l'interprétation d'un programme dépend largement du mode d'énonciation qu'on lui attribue ». (Jost, 1998 :35). Plus tard, l'auteur introduit le terme d' «archigenre », renvoyant au concept d'architexte de Genette (1979), pour introduire l'hypothèse de trois mondes (réel, fictif, ludique) en tant qu'univers de référence de l'énonciation télévisuelle. (Jost, 2005 : 35-39) 
Jost a introduit et définit la notion de genre télévisuel en insistant sur la polyvalence de l'utilisation du terme. Les genres télévisuels sont, selon lui, « au carrefour de logiques diverses », permettant d'informer le téléspectateur, orienter la réception d'un programme et proposer des interprétations, sans oublier les usages stratégiques (économiques, publicitaires, marketing) du genre. (Jost, 1999 : 21-25). Ainsi, en matière d'énonciation télévisuelle, le genre est l'instrument qui permet à la chaîne de situer un programme par rapport aux autres et définir un cadre de réception pour les téléspectateurs. Cette « étiquette générique » (cf. Jost, 1999 et 2005) qu'on attribue à un programme en nommant la catégorie dans laquelle il se range a des répercussions non seulement sur sa compréhension mais aussi sur celle de la structure de l'offre de la chaîne, de son discours identitaire et de marque.

A ce propos, l'on considère comme identité exprimée par un média, son discours explicite ou implicite sur soi, ses contenus, son image, son mode opératoire qui est, donc, forcément réflexif. Cette définition est proche de ce que Jean-Pierre Esquenazi nomme identité discursive d'un média: "l'identité discursive d'un média exprime l'entreprise, son organisation, sa hiérarchie, ses habitudes et aussi sa situation particulière dans le champ médiatique et quelque fois même, [...] dans le champ politique ». (Esquenazi, 2002, p. 149). Par ailleurs, elle s'inspire de la conception d'une chaine de télévision comme responsable de la programmation et, en général, de l'énonciation, ainsi que comme marque (Jost, 2005 :28-33).

Notre hypothèse est que l'on pourrait étudier l'histoire de la télévision d'un pays à travers l'évolution des genres. L'étude d'un genre, en l'occurrence, celui des débats politiques télévisés, se révèle éclairante pour l'essor d'une télévision, si on l'articule avec d'autres éléments contextuels qui structurent les situations que l'on étudie. Pour Eliséo Véron, «la description d'un ensemble de propriétés discursives n'est pertinente que si elle est faite à la lumière d'hypothèses (explicites ou implicites) sur les conditions de production et de consommation des discours » (Véron, 1983: 99). Ces propos traduisent l'idée selon laquelle les discours sociaux, dont une forme d'expression se trouve dans les discours télévisés, ne peuvent être analysés de façon immanente. Adhérant à cette stratégie, il nous semble utile d'étudier l'évolution des programmes politiques à la télévision grecque en s'attardant sur leur contexte de programmation et les mutations dans l'environnement politique et social. A ce sujet, le fait que la télévision grecque a évolué dès ses premiers pas à l'aube de la dictature (1967-1974) et a été établie 
comme institution durant celle-ci est révélateur de son rôle comme instrument d'un pouvoir autoritaire et antidémocratique. Sous le régime des colonels du 21 avril 1967, la télévision a été considérée comme un moyen de propagande, de la même façon que la radio ${ }^{1}$ l'était sous le régime de dictature de Ioannis Metaxas. (Cf., Papathanassopoulos, 1994 : 261).

\section{A. La période du monopole de l'État ${ }^{2}$}

\section{Les années après la dictature (1975-1981)}

Etant donné que chaque système clos développe ses mécanismes répressifs propres qui le protègent, le système oppressif installé lors de la dictature des colonels a été particulièrement résistant en ce qui concerne la télévision. (Kastoras, 1990 : 108-109). En ce sens, on peut entrevoir un prolongement des pratiques autoritaires de la dictature au sein du processus de transition vers la démocratie (Diamandouros, 1984 : 50-71). Ce phénomène indique qu'une dictature ne disparaît pas en laissant derrière elle un 'vide absolu' (Kafetzis, 1998 : 38), puisque la légitimation du régime démocratique rétabli dépend aussi de la 'gestion' du passé autoritaire. ${ }^{3}$ L"intervention gouvernementale sur la radiotélévision grecque et, en particulier, sur les programmes d'information a été constante pour la période qui a suivi la chute des colonels, au point que cette dernière devienne un moyen de diffusion des idées et des décisions de chaque gouvernement. Les journaux télévisés ressemblaient plutôt à des porte-paroles officieux et la censure était omniprésente

1 La naissance de la radiophonie publique a eu lieu en novembre 1935 avec la loi 19/20.11.1935 qui a créé le 'Service d'Emissions Radiophoniques'. Durant l'occupation allemande la radiophonie était totalement contrôlée par les Allemands.

2 La totalité de nos informations sur les émissions diffusées avant 1990 émane essentiellement des sources écrites, telles les archives écrites de la Radiotélévision Hellénique (ERT) et les magazines de télévision (les références bibliographiques mises à part). Nos tentatives d'interprétation et d'explication ont été effectuées donc en l'absence du matériel audiovisuel en question car les archives pour cette période sont inexistantes.

3 Di Palma G., 'Governement Performance : An issue and Three Cases in Search Theory', in G. Pridham, The New Mediterranean Democraties : Regime Transition in Spain, Greece and Portugal, Frank Cass, Londres, 1984, p. 172-187, cité par Kafetzis, 1998. 
et intense. De surcroît, la direction de la radiotélévision fut nommée (et est nommée jusqu'à aujourd'hui) selon ses affinités politiques et fut changée à chaque fois qu'un nouveau parti politique arrivait au pouvoir. Le contrôle imposé par chaque gouvernement, était la raison pour laquelle, depuis l'époque de la restauration de la démocratie, les cadres supérieurs de la direction de la Radiotélévision Grecque changent très souvent. Dans ce contexte, la première émission de débat politique à la télévision hellénique fut, de toute évidence, 'Table Ronde'. Sa programmation a commencé au courant de 1975, peu après le rétablissement du régime démocratique en Grèce. Elle a été suivie par une deuxième émission, intitulée 'Débat Libre', devenue plus tard 'Thèmes et points de vues'. Le choix de la première chaîne de télévision, ERT, pour la diffusion de ces émissions témoigne du statut encore ambigu de la deuxième chaîne publique, YENED ${ }^{1}$, créée sous la dictature par les Services de l'Armée.

Ces émissions étaient présentées par des journalistes divers, car à l'époque la notion du présentateur n'avait pas encore acquis une importance considérable dans la programmation des chaînes. Le fait que les présentateurs des premiers débats télévisés n'étaient pas mentionnés dans le programme de la revue Radiotileorassi, éditée par la Radiotélévision Grecque (ERT) et ne figurent pas dans les archives écrites de la télévision publique va dans le même sens. De même, le fait qu'à leurs débuts, le jour de diffusion dans la semaine soit variable, fait preuve d'une grille de programmation souple, propre à l'émergence d'un genre qui faisait ses premiers pas et cherchait à se définir.

Le seul élément stable permettant l'identification de ces émissions et leur repérage dans cette programmation quelque peu primitive est le titre. Ce dernier, était suffisamment explicite pour suggérer au moins le genre du programme diffusé, constituant le principal point de repère. Par ailleurs, un examen des titres des premières émissions sur l'écran laisse entrevoir une volonté d'initier le dialogue en tant que pratique politique et de rompre avec les dispositifs antérieurs de monologue. Ceci présente un élément à ne pas perdre de vue, d'autant plus que le choix d'un titre clair et explicite ne fut pas toujours le cas pour les émissions postérieures. Le rapport donc entre la construction d'une grille et l'appellation des programmes télévisuels est étroit et particulièrement

1 En grec: 'YENE', qui est l'abréviation de 'Service des Forces Militaires Grecques'. 
intéressant pour la compréhension de la logique structurante et fonctionnelle d'une chaîne de télévision.

Un autre élément de programmation est le court format des débats qui n'excédait pas les trente minutes environ; de plus, leur diffusion était programmée toujours après 21 heures. Plus significatif encore est le fait que les émissions en question avaient presque toujours la forme de débat entre plusieurs invités. Cela pourrait être interprété comme une sorte de prudence, le nombre d'invités fonctionnant en tant qu'amortisseur des affrontements directs sur l'écran. Comme le note Eric Darras, les rencontres télévisuelles des journalistes et des hommes politiques, ne doivent pas être considérées comme 'naturelles'; tout au contraire, elles ont été historiquement constituées (Darras, 1997 : 11). Cette hypothèse est renforcée par le statut des invités choisis pour y participer. En effet, la plupart du temps, il s'agit des invités qui jouent, certes, un rôle dans le processus politique ou civique, mais qui ne font pas partie des acteurs de premier plan, comme serait, par exemple, le cas des ministres. De plus, l'idée d'interviewer le premier ministre ou le Président de la République dans le cadre d'une émission de ce type n'était même pas envisageable car le dialogue était exclu des pratiques de communication politique des années 70 . En outre, les thèmes des débats portaient sur des sujets politiques au sens large du terme, comprenant des thèmes sociaux, économiques et civiques. Cet aspect est conforme à notre hypothèse, à savoir que l'idée de débat politique en tant que genre pur et indemne existe dans l'autodéfinition et l'autoconfiguration de ces émissions qui sont soumises à une classification. Cette remarque à propos du genre 'débat politique', est intéressante pour se rendre compte de l'historicité des questions sur les genres qui dépassent les clivages construits par une ère de télévision publique et l'avènement d'une ère de télévision privée ; cela ne nous empêche pas, toutefois, de reconnaître la dynamique créée par ces phénomènes.

Néanmoins, le caractère politique de 'Table Ronde' et de 'Débat Libre' n'est pas mis en question et cela pour des raisons diverses : ces émissions étaient produites par la direction des programmes d'information et par le service d'information politique. De surcroît, elles étaient les premières, ensemble avec le journal télévisé, à subir les restrictions des dirigeants gouvernementaux. A ce propos, l'irrégularité des parutions peut aussi être envisagée comme résultat direct ou indirect d'un contrôle et d'une censure gouvernementale pesante. Il n'est pas un hasard si jusqu'à nos jours, la durée de vie des émissions politiques 
(ainsi que d'autres programmes radiotélévisuels) dans le secteur public, correspond souvent à la durée de vie du gouvernement.

\section{Le débat au sein de la télévision des socialistes (1981-1989)}

En effet, c'est ce que l'on remarque dès l'avènement du PASOK au gouvernement en 1981. Les émissions mentionnées ci-dessus cessent d'être diffusées et leur place est prise par 'Cartes sur Table' (1982-1989). En 1987, une autre émission apparaît aussi, s'intitulant 'Face à la Presse'. Ces émissions bimensuelles ont été diffusées en alternance et font apparaître des caractéristiques différentes de celles de leurs ancêtres. On remarque l'évolution et l'affinement de certains traits définitoires d'un genre émergent à la télévision grecque. Parmi les observations les plus évidentes que l'on puisse faire, la première serait de remarquer que le présentateur, en tant que figure centrale de l'émission, commence progressivement à prendre forme. En effet, les deux émissions en question, étaient présentées en alternance par deux journalistes. En même temps, la durée des émissions ne cesse pas de s'étendre.

Par ailleurs, l'émission 'Cartes sur Table' avait inclus dans son dispositif la présence en studio d'une quinzaine de personnes représentant la population. Le public sur le plateau avait le droit de poser des questions aux invités politiques. Ceci fut un cas presque exceptionnel dans la télévision hellénique, en ce qui concerne les émissions politiques. Ce type de dispositif fut peu renouvelé, même durant les années de la télévision privée. Cependant, à l'ère du premier gouvernement socialiste dans l'histoire politique du pays, un tel dispositif était conforme aux attentes sociales dans tous les domaines. En ce sens, la présence d'un public actif et participant devrait être perçue comme un indice démocratique dans le processus politique, tel qu'il était représenté à la télévision. Toutefois, ce dispositif d'émission a été très rapidement abandonné et n'a connu ni une évolution dynamique ni une utilisation régulière à travers les années. A ce sujet, le recours au public seulement à des occasions sporadiques et isolées, confirme de façon plus marquante l'absence d'une parole civile médiatisée. ${ }^{1}$

1 A titre d'exemple, on pourrait mentionner les émissions 'Profil', 'La Pomme de discorde' (1995-97), 'Face à Face', qui ont inclus un public sur le plateau, condamné, cependant, au silence et réduit à un dispositif scénique. Egalement, pendant une courte période au milieu des années 90, l'émission 'L'Heure de Vérité' accordait la 
En revanche, en ce qui concerne l'évolution des invités, leur choix va dans le sens inverse. Progressivement, les dialogues et les débats entre des journalistes et des hommes politiques directement liés au pouvoir exécutif deviennent la règle d'une émission politique. La présence sur les plateaux des ministres du gouvernement du PASOK et des représentants des autres partis politiques qui acceptaient d'être interrogés par des journalistes, représenta un nouveau mode de communication et de médiation politique. Les pratiques suivies par le parti socialiste et le passage de l'allocution au dialogue se voulaient en opposition avec les méthodes jugées autoritaires du gouvernement précédent de Nea Dimocratia. (Cf. aussi Psilla, 1990). Toutefois, il ne faudrait pas perdre de vue les limites restreintes de cette 'liberté' d'information indépendante, à savoir le traitement privilégié des positions gouvernementales et le contrôle constant sur les médias audiovisuels. Vu sous cet angle et en ce qui concerne la télévision, il y a bien eu une continuité dans le comportement des gouvernements après la dictature.

Les émissions 'Cartes sur table' et 'Face à la presse', cesseront leur diffusion avec la chute des socialistes du pouvoir. Dès l'installation du nouveau gouvernement de Nea Dimocratia d'autres émissions sont apparues, puis expirées avec lui, comme les émissions 'Seconde Edition' (1991-93) et 'Incisions' (1991-94). Pour d'autres, comme l'émission 'Profil' (1990-2004), la naissance de la télévision privée a créé une alternative et une possibilité de survie et d'évolution fructueuse. Cette tradition paradoxale d'un status quo aussi officieux qu'incontestable dans le contexte d'une société qui déclare son attachement aux idéaux démocratiques, profite d'une continuité remarquable. En effet, les élections de 1993 qui ont amené à nouveau le PASOK au pouvoir, ont été suivies par un changement respectif dans les services d'information télévisuelle. En matière de programmes politiques, certaines émissions comme par exemple, 'Dans l'ensemble des choses' et 'Ligne Finale' ont trouvé leur place dans la programmation de la télévision publique. Avec ces remarques, nous n'entendons pas insinuer l'incompétence des journalistes-présentateurs desdites émissions, ni sous-estimer leur apport dans la formation du paysage audiovisuel hellénique. Il s'agit, plutôt,

parole au public vers la fin de l'émission. Ainsi, pour quelques minutes, des citoyens présents sur le plateau pouvaient poser des questions aux invités politiques. Cette tentative ne fut que rarement renouvelée au sein des émissions de débat politique. (Vovou, 2002). 
de signaler ce qui constitue un fait dans la pratique politique en Grèce, qui trouve ses répercussions dans différents aspects de la société.

\section{Les débats à partir des années 90}

Dès leur création, les deux premières chaînes privées, Mega et Antenna, ${ }^{1}$ ont constitué des journaux télévisés et des émissions d'actualité politique concurrentiels. Les interviews politiques diffusées par les médias audiovisuels sont apparues avec la création de la radiophonie privée et ont pris une ampleur considérable durant les premières années de création des chaînes privées de télévision. ${ }^{2}$ Avec le début des chaînes privées, deux émissions intitulées l'une 'Altercations', donnant lieu par la suite à l'émission 'Face à Face', et l'autre 'Profil' apparaissent sur le petit écran. La première était diffusée sur Mega et la deuxième sur la première chaîne publique ET1 (anciennement appelée ERT) jusqu'en avril 92. A partir de cette date, 'Profil' se déplace sur la chaîne privée Antenna. L'interview 'face à face', enrichie selon les cas des duplex et des interventions d'invités supplémentaires, était le modèle de base établi par les deux émissions. Durant une longue période d'environ trois ans ces dernières étaient considérées comme les émissions- 'phares' de la nouvelle ère télévisuelle en Grèce.

La création et l'établissement d'autres chaînes privées ont permis aux émissions d'interview et de débat de connaître une évolution remarquable. Dorénavant, toutes les chaînes -y compris celles du service public- vont inclure une, deux, voire plusieurs émissions politiques dans leur programmation hebdomadaire. Malgré l'existence occasionnelle des interviews politiques durant le monopole de l'ERT, nous pouvons considérer que l'interview politique télévisée en tant que genre journalistique légitime est relativement récente en Grèce. Il a développé des structures solides et élaborées au sein de la télévision privée et a connu un accroissement sans précédent. Dans une ambiance où télévision privée rimait avec liberté, l'interview politique face à face a été privilé-

1 Mega commence officiellement sa diffusion le 20 novembre 1989 et Antenna le 31 décembre 1989, le soir du réveillon.

2 A ce sujet, une enquête datant de janvier 1993 et concernant les années 1991 et 1992 démontrait que le nombre d'interviews données chaque mois pour l'ensemble de la presse écrite et audiovisuelle atteignait les quatre mille. Pourtant le nombre de personnes interviewées restait relativement limité (Publiée dans le journal de dimanche 'Kyriakatiki Eleytherotypia' du 24.01.93). 
giée pendant les premières années qui ont suivi la déréglementation des ondes hertziennes. Pour la première fois les hommes politiques étaient à la disposition des journalistes, en face d'eux, pour répondre à leurs questions. Le 'face à face' présenta la garantie d'un affrontement direct à travers lequel la vérité surgirait. Après le règne des émissions d'interview politique, les émissions de débat prennent en grande partie le relais, mais dans une optique légèrement différente de celle rencontrée dans les années 70 qui consistait à amortir les affrontements. Le débat à plusieurs, dirigé par un meneur de jeu incarné dans l'image du présentateur, a été considéré comme un modèle dialectique plus propice pour la production ou l'accouchement de la vérité. Les nombreuses possibilités de mise en scène et de mise en spectacle sont devenues impératives pour répondre à la demande d'audience et à la concurrence ; par conséquent, elles n'allaient pas rester inexplorées.

\section{Rigidification des grilles et identité des chaînes}

En même temps, la diffusion irrégulière cède la place peu à peu à une rigidification des cases horaires. L'inclusion des émissions d'interview et de débat dans la grille des programmes des chaînes devient régulière et s'inscrit dans la construction de l'identité des chaînes. Ainsi, la grille de programmation commence, par ailleurs, à se rigidifier aussi bien horizontalement que verticalement. Cela répond à un besoin des chaînes " [d'] assurer aux annonceurs un public prévisible quantitativement et qualitativement stable». (Jost, $1995: 52$ )

L'étude du contexte pour les programmes télévisuels est essentielle pour leur compréhension. C'est la grille des programmes qui les situe dans le flux temporel de la diffusion et qui tente de se calquer sur la vie quotidienne. Dans ce contexte donc, les émissions politiques des années 90 deviennent des programmes réguliers, profitant d'un jour de diffusion plus ou moins stable. La plupart d'entre elles ont une périodicité hebdomadaire, ce qui signifie qu'elles font partie intégrante de la stratégie des chaînes et de la construction de leur identité. De ce fait, leur statut gagne en légitimité par rapport aux émissions de débat à l'ère de la télévision publique. La programmation hebdomadaire leur permet de construire une présence permanente et significative aux yeux des téléspectateurs, s'insérant dans la temporalité télévisuelle et sociale. Le jour de diffusion stable contribue à ce résultat, établissant un 'rendezvous' avec une partie du public dans le même registre que les autres programmes télévisuels. En d'autres termes, les débats télévisés s'in- 
tègrent dans le discours général que les chaînes tiennent à leur public. Ce discours est fondé sur la cohérence, la régularité et la continuité. Ce sont là des éléments qui rendent lisible la stratégie énonciative que la chaîne propose explicitement et implicitement à ses téléspectateurs. (voir aussi Semprini, 1996 : 175-176)

La variabilité parfois observée dans la programmation du service public s'explique par une absence de stratégie rigoureuse que les chaînes privées ne peuvent pas se permettre. Bien qu'elle soit rencontrée de moins en moins fréquemment, cela a entraîné des problèmes dans la construction d'une identité claire et cohérente pour l'ERT. De même, ce phénomène a des conséquences néfastes sur la fidélisation du public, ce qui est traduit par une audience extrêmement faible. En effet, les deux types de télévision, d'une part ET1, ET2 et ET3 et d'autre part, les chaînes privées, obéissent à des logiques différentes. Les premières reposent sur un financement par impôt, inclus dans la facture de l'électricité, tandis que les deuxièmes sont plus intéressées par le consommateur potentiel incarné dans le téléspectateur, car leur financement par la publicité est impératif. Dans la mesure où la publicité est considérée comme moyen de financement supplémentaire pour la télévision publique aussi, les logiques structurantes des deux systèmes se rapprochent. Cela est essentiellement traduit en termes de programmation fidélisante et beaucoup moins en termes de programmes offerts. A titre d'exemple, certains programmes télévisuels jugés de qualité médiocre, comme les reality shows, ne figurent pas dans les grilles des chaînes publiques.

Toutefois, cette perspective de programmation ne doit pas se confondre avec la construction d'un flux continu. La conception traditionnelle des programmes télévisuels comme œuvres uniques coexiste avec la notion de l'offre continue de programmes. Ainsi, la programmation des émissions politiques se fait, la plupart du temps dans la semaine. En général, samedi et dimanche ne sont pas considérés comme des jours propices pour des débats politiques d'actualité et la fin de la semaine est a priori exclue de la mission informative ; de cette façon, le divertissement et la fiction lui sont davantage réservés. La programmation de la quasi-totalité des débats politiques à la télévision grecque se fait donc pendant la semaine et à des horaires tardifs. Presque toutes les émissions sont programmées après 22 heures et peuvent durer après minuit, voire une heure du matin. Pour la majorité des chaînes grecques, la zone du prime-time entre 20 heures et 23 heures est occupée pendant la semaine, par le journal télévisé en début de soirée et, ensuite, par des 
séries télévisées. Les chaînes du service public, fidèles à une mission informative plus marquée, programment les émissions politiques vers 22 heures. En revanche, les chaînes privées commencent la diffusion de ces émissions entre 23 heures et $23 \mathrm{~h} 30$. Toutefois, il serait intéressant de savoir que presque toutes les émissions politiques programmées tard dans la soirée sont précédées par des fictions, à savoir des séries télévisées et des feuilletons à caractère souvent comique. Cette observation est révélatrice du registre de disponibilité réceptive des téléspectateurs, contradictoire, à première vue, avec le genre et le ton du programme qui suit. La continuité tonale ou thématique (en ce qui concerne certains invités par exemple) qui s'observe parfois dans les émissions de débat politique découle directement de la stratégie de programmation des chaînes. (Vovou, 2000).

En outre, toujours pour la période après 1990, la durée des émissions s'étend considérablement. Elles quittent définitivement les formats courts d'une demi-heure pour atteindre la durée d'une heure, d'une heure et demie, voire parfois plus. D'une part, la combinaison de dispositifs divers, tels que les duplex en direct et les reportages enregistrés intercalés entre les séquences dialogales et, d'autre part, l'augmentation impressionnante du nombre des invités de plateau, expliquent cette évolution. Dans ce contexte, nous observerons que l'extension de la durée des débats, ne correspond pas nécessairement à un plus grand temps de parole des participants.

\section{L' « identité » des présentateurs et des invités}

Une autre caractéristique de cette nouvelle vague d'émissions politiques concerne l'identification d'une émission à son présentateur. Le statut du présentateur qui a commencé à avoir une substance autonome au cours des années 80 , se définit de façon claire à l'ère de la télévision privée des années 90 . Le présentateur devient la figure dominante des émissions de débat politique. Pour se rendre compte de l'ampleur du phénomène il suffit de parcourir certaines dénominations qui reprennent les noms de leur présentateur comme par exemple 'Anna dans le pays des merveilles'(1991-93), 'Ellispontos'(1995-96), 'Extremismi'(1997-98) et 'A travers les yeux d'Elli'(1999-...). De plus, certains génériques d'émissions au sein desquels le visage du présentateur figure en gros plan et dont la coiffure et les soins vestimentaires apparaissant sur les titres de la fin sont aussi révélateurs. En d'autres termes, la fidélisation du téléspectateur n'est pas uniquement obtenue 
par la stabilité et la régularité de la programmation, mais aussi par le présentateur. Ce dernier fonctionne comme un point de repère central des émissions de débats, au sein desquels défile un nombre important de personnages politiques. Les mutations de ces programmes en deuxième moitié des années 90 et au début des années 2000, en relation avec la multiplication des chaînes et le recul du caractère purement politique des débats, trouvent le présentateur incarner la marque du programme, plus encore que celle de la chaîne. Le fait que les émissions en question suivent les journalistes/présentateurs dans leur changement de chaînes, en subissant quelques modifications de dispositif, de format et, parfois, du titre (surtout pour la période la plus récente), témoigne de cette situation. Ceci a été le cas, par exemple, pour l'émission Profil qui depuis sa création a été diffusée sur la chaîne publique ET1, puis, sous le même titre, sur différentes chaînes privées (Antenna, Star, Skaï, Alter...) avant que son présentateur Panos Panagiotopoulos ne devienne débuté, puis ministre. De même, l'on pourrait citer l'émission de Face à Face de Nikos Hatzinikolaou, diffusée sur Mega channel, devenue Opinions différentes quand le journaliste passe sur la chaîne Alpha; dans le même registre, l'émission Ellispontos d'Elli Staï, diffusée sur Mega channel devient A travers les yeux d'Elli sur Antenna et L'Heure de Vérité de Yannis Pretenderis, diffusée sur Antenna, change en Renversement, sur Mega. Même dans le cas où le concept des émissions présente des différences structurales par rapport aux diffusions antérieures, au point de les considérer comme des émissions nouvelles ou comme des variantes, et malgré le fait qu'elles soient produites en interne par les chaînes, elles cessent leur diffusion, ne faisant pas appel à de nouveaux présentateurs (ce qui peut, au contraire, arriver pour certains magazines télé matinaux, par exemple). Ceci est significatif du rôle que les présentateurs jouent en tant que figures symboliques participant à la construction de l'image de marque des chaînes.

Dans le contexte d'une comparaison avec les premiers débats des années 70, nous observons aussi d'autres changements formels. A ce sujet, l'interview 'face à face' avec un homme politique est considérée comme trop dangereuse et n'est pas envisagée, en général, à la télévision jusqu'à la fin des années 80 . Après cette date, les 'face à face' avec des hommes politiques font leur apparition à la télévision grecque et auront une évolution considérable. Par ailleurs, le terme d'invité 'spécialiste' (journaliste spécialisé, professionnel, homme politique ou acteur social) remplace celui des 'personnalités connues' des premiers débats. Nous constatons que les personnalités célèbres invitées sur 
les plateaux ne sont pas une nouveauté à la télévision, même si elles répondent à un besoin différent ; durant les premiers pas des émissions de débat ce type d'invités représentait une solution 'anodine' qui ne soulevait pas de controverse face au contrôle du pouvoir politique. En revanche, le recours au 'face à face' de nos jours répond plutôt à des besoins d'atteindre une audience maximale, profitant de la notoriété de l'invité.

\section{Les « empreintes » de forme et de style}

En dernier lieu, le cas des émissions issues de modèles français, par exemple 'L'Heure de Vérité' (1995-2000), 'Sept+Sept'(1994-1999) et 'Ellispontos' désigne aussi un phénomène particulier. Dans la mesure où ces émissions dépassent le stade d'un simple mimétisme pour s'inscrire dans les données de la télévision et de la société grecques, elles méritent qu'on s'y attarde un peu. Ces trois exemples révèlent de deux types d'emprunts concernant les émissions politiques: le premier consiste en l'emprunt d'une partie du dispositif de l'émission prototype (scénographie, construction et rubriques de l'émission). L'émission grecque 'L'Heure de Vérité', inspirée de l'émission française homonyme et ' $7+7$ ', inspirée de ' $7 / 7$ ' rentrent dans cette catégorie. Le deuxième type, très intéressant, consiste en la tentative d'imiter et de s'approprier l' "âme » de l'émission, à travers l'image du présentateur ; c'est le cas de l'émission 'Ellispontos' et de sa présentatrice Elli Stai qui a une admiration considérable pour Anne Sinclair. Dans les deux cas, l'éloignement du prototype se fait remarquer progressivement mais très rapidement (Vovou, 2003). La construction d'une figure de présentateur emblématique selon les critères de la société grecque est un des éléments centraux dans la cristallisation du concept des ces émissions qui se fait indépendamment du modèle d'origine.

Vues sous ce prisme, les émissions issues des modèles français témoignent de deux tendances opposées qui dominent nos sociétés contemporaines, à savoir d'une part la 'mondialisation' et d'autre part la résistance d'identités nationales. La dialectique qui naît est la circulation des modèles et leur assimilation selon les conventions symboliques fonctionnant dans chaque pays. De cette façon, les émissions grecques qui se sont inspirées des modèles étrangers tracent leur propre chemin, adaptant les différents traits formels pour pouvoir répondre à diverses exigences. Ces dernières concernent le paysage politique, le contexte économique des chaînes et celui de la programmation, l'horizon d'at- 
tentes des téléspectateurs, la personnalité du présentateur, mais aussi les structures psychologiques de la société. Ces aspects dessinent des paramètres qui diffèrent des émissions prototypes. En définitive, à travers la circulation et l'assimilation des concepts et des modèles étrangers, on voit apparaître des émissions de débat grecques tout à fait différentes et autonomes. En ce sens, les programmes venus d'ailleurs constituent des sources d'images de l'autre qu'on utilise afin de construire sa propre image, selon une démarche qui consiste à découvrir soi à travers le visage de l'autre.

\section{Pour une mise en perspective diachronique}

Ce détour par les émissions inspirées des débats français nous permet d'énoncer notre idée centrale qui consiste à penser que la télévision est l'expression d'une société, inscrite dans une historicité précise, tout en suivant des logiques économiques sur un plan international. A travers l'étude du contexte de programmation des débats politiques nous avons voulu mettre en évidence la complexité des paramètres à prendre en considération quant à l'énonciation des règles générales appliquées à la télévision. Notre étude montre ainsi, qu'il n'y a pas de périodisations complètement étanches, avec des modes de fonctionnement propres, de la même façon qu'il n'y a pas d'évolution linéaire. Ce qu'il y a, en revanche, est l'interférence d'éléments divers dans la composition des phénomènes télévisuels.

En l'occurrence, dans notre présentation, un certain nombre de remarques vont à l'encontre de l'idée qu'il existerait une paléo-télévision et une néo-télévision, figeant l'histoire de ce média en deux époques distinctes constituées en complète opposition. ${ }^{1}$ En ce qui concerne plus particulièrement la télévision grecque, par exemple, la bonne raison d'être de la mission éducative de la télévision est mise en cause dès les années 70, par des voies en provenance du milieu artistique, mais aussi par le public. ${ }^{2}$ Par ailleurs, les logiques divergentes en

1 Parue, initialement, dans la presse italienne, cette thèse énoncé par Umberto Eco a bénéficié d'une grande popularité, puis elle a été largement critiquée. Pour l'édition française voir Eco Umberto «TV : La transparence perdue », in La guerre du faux, Grasset, 1985 et Casetti Francesco et Roger Odin, 'De la paléo à la néo-télévision', in Télévisions Mutations, Communications n51, Le Seuil, 1990, pp. 9-26.

2 Dans l'esprit quasi uniforme de l'époque où éducation civile rimait avec mission télévisuelle il y avait malgré tout des voix qui échappaient à cette logique. Par exemple 
termes de programmation, suivies par le service public de la radiotélévision et les chaînes privées, vont au-delà d'une périodisation chronologique et ne peuvent être expliquées uniquement en ses termes. Par exemple, en ce qui concerne l'existence des genres 'purs' à la paléotélévision et la pratique du mélange de genres par la néo-télévision, la diversité des sujets traités dans les émissions dites politiques dans les années 70 rend fragile ce type de discours et trouve sa raison dans les marges étroites d'expression politique. De même, la présence sur les plateaux des personnalités célèbres n'est pas un phénomène nouveau mais existe aussi avant l'avènement de la télévision privée pour des raisons différentes, à savoir l'impossibilité d'interroger les dirigeants politiques. Au courant des années 90 ce choix de diversification des thèmes et des invités en dehors du champ politique stricto sensu, renvoyant partiellement à des glissements normatifs du genre, est soustendu par la stratégie de programmation et d'offre des programmes par les chaînes, privilégiant la dilution de l'objectif politique dans celui de débat social, voire 'mondain' ou 'people'. Le processus de rigidification des grilles, certes à travers la naissance d'une figure présentatrice dominante mais contre l'idée d'un flux continu de programmation, va dans le même sens de notre démonstration, à savoir celui d'une évolution à rebondissements, avec une coexistence des formes et des schémas, malgré les logiques politiques ou économiques différentes, selon les périodes, qui les sous-tendent. En dernier lieu, le processus de morcellement du marché audiovisuel grec qui s'est accentué à partir de la deuxième moitié des années 90 et la multiplication des chaînes privées remodèle le paysage audiovisuel, indiquant des stratégies différentes de la part des entreprises médiatiques en matière d'offre de programmes. Ces exemples ne font que mettre l'accent sur l'importance des éléments

\footnotetext{
Manos Hatzidakis, un des plus grands compositeurs grecs, qui était à l'époque au poste du 'Directeur Général Remplaçant' de la scène lyrique nationale, affirmait déjà en 1974 : « Ces conceptions concernant l'éducation du public sont antidémocratiques et ontsouvent desintentions suspectes ». (Revue Radiotileorassi, 24-30novembre 1974). De même, la primauté du rôle éducatif de la télévision n'était pas forcement une opinion partagée par le public. Déjà en 1975, un sondage de la revue Radiotileorassi, montre que les téléspectateurs étaient fortement partagés en ce qui concerne les émissions éducatives : 24\% du public aimerait que leur nombre reste inchangé, 26\% souhaiterait qu'elles diminuent et $17 \%$ souhaiterait une augmentation. Cependant, en ce qui concerne les émissions divertissantes les opinions étaient plus convergentes : $65 \%$ du public voudrait voir plus d'émissions divertissantes. (Radiotileorassi, 12-18 janvier 1975).
} 
extra-télévisuels à prendre en considération, afin d'essayer de reconstituer les fragments de l'identité culturelle d'une société, toujours en évolution, dont la télévision fait partie.

\section{Références bibliographiques}

- DARRAS Eric, 'Les bienséances de l'échange politique', in 'Télévision et Politique', Politix $n^{\circ} 37$, L'Harmattan, 1er trimestre 1997, p. 9-24.

- DIAMANDOUROS Nikiforos, 'Transition to and Consolidation of Democratic Politics in Greece 1974-1983 : A Tentative Assesment', in G. Pridham (Sous la Direction de), The New Mediterranean Democraties : Regime Transition in Spain, Greece and Portugal, Frank Cass, Londres, 1984.

- ESQUENAZI Jean-Pierre, L'écriture de l'actualité, Presse Universitaires de Grenoble, 2002.

- GENETTE Gérard, Introduction à l'architexte, in G. Genette (dir.), Théorie des Genres, Seuil, 1986.

- JOST François, - 'Téléspectateurs modèles et modèles téléspectateurs', in La télévision et ses téléspectateurs/Champs Visuels, L'Harmattan, 1995, pp. 49-62.

- 'Quant y a-t-il énonciation télévisuelle ?', in J. Bourdon, F. Jost (dir.), Penser la télévision, INA/Nathan, 1998, pp. 29-58.

- Introduction à l'analyse de la télévision, Paris, Ellipses, 1999.

- Comprendre la télévision, Paris, Armand Colin, 2005.

- KAFETZIS Panayiotis, 'La dictature dans la politique de masse de la IIIème République Hellénique. Une approche empirique', in Revue Hellénique de Science Politique, $\mathrm{n}^{\circ}$ 12, novembre 1998, Themelio, pp. 35-56.

- KASTORAS Stavros, Médias Audiovisuels: Sciences de la Communication, Papazissis, 1990.

- PAPATHANASSOPOULOS Stylianos, En libérant la Télévision, Kastaniotis, 1994.

- PSILLA Marianna, La communication politique en Grèce : le cas de la télévision, Thèse de Doctorat, Université Paris I, 1990.

- SEMPRINI Andrea, Analyser la Communication, Comment analyser les images, les médias, la publicité, coll. Champs Visuels, L'Harmattan, 1996. 
- SCHOLES Robert, 'Les modes de la fiction', in Gérard Genette (dir.), Théorie des Genres, Seuil, 1986.

- VERON Eliséo, 'Il est là, je le vois, il me parle', in Communications $\mathrm{n}^{\circ} 38$, Seuil, 1983.

- VOVOU Ioanna, - La Démocratie à l'ère de la télévision. Les débats politiques à la télévision hellénique, Thèse de Doctorat, Université Paris III-Sorbonne Nouvelle, 2000.

- «Formats d'émissions politiques à la télévision : le modèle français de débat en Grèce », revue Médiamorphoses n7, INA, 2003, pp. 83-88.

- «Le public 'perdu de vue' ou comment le peuple est représenté dans les émissions politiques grecques », Mésogeios $n^{\circ} 16$, éditions Hêrodotos (octobre 2002), pp.155-176.

\section{Sources}

- Archives écrites et audiovisuelles de la radiotélévision hellénique

- Archives audiovisuelles des chaînes privées.

- Radotileorassi, revue éditée par la Radiotélévision Grecque (ERT) depuis 1939. 